\title{
ANALYTICAL AND NUMERICAL INVESTIGATION ON THE DUFFING OSCILATOR SUBJECTED TO A POLYHARMONIC FORCE EXCITATION
}

\author{
SvetLin Stoyanov \\ Department of Technical Mechanics, University of Ruse, \\ 8 Studentska Str, POB 7017, Ruse, Bulgaria, \\ e-mail: SStoyanov@uni-ruse.bg
}

[Received 22 September 2014. Accepted 09 March 2015]

\begin{abstract}
An analytical solution for a specific case of the forced Duffing oscillator is proposed. The excitation force contains two harmonics with significant difference frequencies. This case corresponds to a presence of a defect in the machinery and is in the art of the machinery vibration diagnostics. The results obtained show an amplitude modulation. Therefore, the presence of an amplitude modulation in the vibration signal may be used as an indicator for a malfunction. Analytical solution derived clarifies how the amplitude modulation occurs. Also, a numerical solution is realized and compared with the analytical one. For this, the Duffing equation is solved numerically and then, the spectrograms of vibrations are obtained through a Discrete-time Fourier Transform.

KEY WORDS: Machine vibration, monitoring, incipient faults, early detection, vibration diagnostic, nonlinear vibrations, Duffing equation, Duffung oscillator, polyharmonic force excitation, analytical solution, numerical solution, spectrograms, discrete-time Fourier transform.
\end{abstract}

\section{Introduction}

Machine monitoring and early detection of incipient faults gives an ability to avoid human casualties and significant financial losses. Machine monitoring aims to survey the machine health at critical locations, e.g. gears and bearings, and predict a future failure. A scheduled stop for maintenance can be made and the damaged element can be replaced at a certain stage of defect progress. Thus, the production runs without unexpected delays. Machine vibration monitoring uses the so called signature analysis, i.e. the characteristic vibration signature of the monitored machine element is investigated. To obtain such signatures, it is needed nonlinear models and advanced methods to 
be used like Finite Element Method [1, 2, 3, 4], Hilbert Transform [5, 6, 7, 8, $9,10]$, and Wavelet analysis [11, 12, 13, 14, 15].

This work gets a simple and basal fact, and builds on it a composition of traditional analytical approach and advanced numerical methods for solving of differential equations and signal processing. The mentioned sample and basal fact is that when machinery has a defect, frequently this results in a polyharmonic force excitation acting on the supports. The harmonics of this force have significant difference in the frequencies. For an example, a gear with a crack in one tooth generates a force excitation with two components: one with higher frequency that occurs when every healthy tooth goes into a contact, and another one with lower frequency only when the tooth with crack goes into a contact. The most appropriate is modelling the stiffness with a cubic function of the displacement [16], if we take in the account nonlinearity of the supports (like bearings and housings). There is also a point mass and this becomes the good known Duffing oscillator [17, 18].

The Duffing oscillator is comprehensively investigated for many cases like: free vibrations [17, 18]; a single harmonic force excitation [17, 18, 19, 20]; a force excitation that contains two harmonics with equal [21] or similar [22] frequencies; an excitation that contains a harmonic force and a noise [23]; and a piece-wise force excitation. In [23], the application of weak signal detection based on chaotic oscillator is studied. Results obtained show that the chaotic oscillator can detect weak signal which is submerged in the background of large noise.

For a single harmonic force excitation, the Duffing oscillator is described by equation:

$$
\ddot{x}+2 \gamma \dot{x}+\alpha x+\beta x^{3}=F \cos (p t+\varphi),
$$

and has a following solution $[17,18,19,20]$ :

$$
\begin{aligned}
\text { (2) } x=\frac{\alpha A+\frac{3}{4} \beta A^{3}-F \cos (\phi)}{p^{2}} \cos (p t) \\
\quad-\frac{2 \gamma p A-F \sin (\phi)}{p^{2}} \sin (p t)+\frac{1}{36} \frac{\beta A^{3}}{p^{2}} \cos (3 p t) .
\end{aligned}
$$

This solution contains two harmonics: one with frequency $p$, and another with frequency $3 p$. We need to find what will be the difference when the excitation force is polyharmonic as these correspond to presence of a defect in machinery. 
The objective of the work presented in this research is to obtain the characteristic vibration signature of polyharmonic force with significant difference in the frequencies of the harmonics, when it acts on a machine element with nonlinear stiffness. This work would be beneficial for machinery vibration diagnostics, as this may give a way to recognize a problem in the health of the machine.

\section{Mechanical and mathematical model of the investigated} system

The mechanical model of the investigated system is sown in Fig. 1 [17, $18,19,20,21,22,23]$. This is discrete model with one degree of freedom. The model parameters are: a point mass $m_{0}$, a multiplier $\alpha_{0}$ for linear stiffness, a multiplier $\beta_{0}$ for cubic stiffness, and a coefficient of linear viscous friction $\gamma_{0}$.

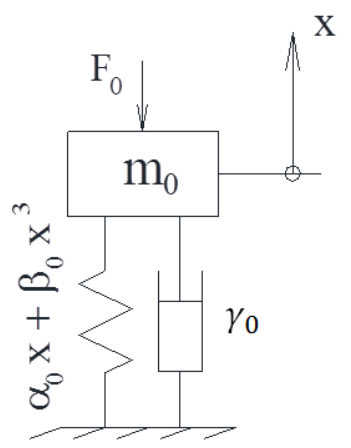

Fig. 1. Mechanical model of the investigated system

The corresponding mathematical model is commonly known $[17,18,19$, 20, 21, 22, 23]. It is the Duffing equation and already has been given by Eq. (1). Unlike those investigations, in this work the excitation force consists of two harmonics with significantly different frequencies:

$$
m \ddot{x}+\gamma_{0} \dot{x}+\alpha_{0} x+\beta_{0} x^{3}=F_{1_{0}} \cos \left(p t+\varphi_{1}\right)+F_{2_{0}} \cos \left(n p t+\varphi_{2}\right) .
$$

In this equation, $n$ is multiplier which takes into the account the differences between the frequencies of the harmonics. Also, every harmonic possesses its own amplitude $F$ and phase $\phi$. One can write:

$$
\ddot{x}+2 \gamma \dot{x}+\alpha x+\beta x^{3}=F_{1} \cos \left(p t+\varphi_{1}\right)+F_{2} \cos \left(n p t+\varphi_{2}\right),
$$


when:

$$
F_{1}=\frac{F_{1_{0}}}{m} ;
$$

$$
F_{2}=\frac{F_{2_{0}}}{m} ;
$$

$$
\gamma=\frac{\gamma_{0}}{2 m}
$$

$$
\alpha=\frac{\alpha_{0}}{m} ;
$$

$$
\beta=\frac{\beta_{0}}{m} .
$$

In this work, Eq. (4) is solved analytically and numerically. Then, the results are compared and conclusions are derived.

To be suitable to solving analytically, two simplifications are made:

- the force acting on the supports is applied as an external excitation force. Actually, it is an internal force and depends simultaneously on the defect and the form of the supports parameters;

- this excitation force is constructed by only two harmonics. It is needed more harmonics in real machinery.

\section{Analytical solution}

By applying an elementary trigonometric relation, one can obtain form Eq. (4):

$$
\begin{aligned}
\ddot{x}+2 \gamma \dot{x}+\alpha x+\beta x^{3}=F_{1}[ & \left.\cos (p t) \cos \left(\varphi_{1}\right)-\sin (p t) \sin \left(\varphi_{1}\right)\right] \\
& +F_{2}\left[\cos (n p t) \cos \left(\varphi_{2}\right)-\sin (n p t) \sin \left(\varphi_{2}\right)\right] .
\end{aligned}
$$

The next step is to make the following assignments:

$$
\begin{aligned}
& C_{1}=F_{1} \cos \left(\phi_{1}\right) ; \quad S_{1}=F_{1} \sin \left(\phi_{1}\right), \\
& C_{2}=F_{2} \cos \left(\phi_{2}\right) ; \quad S_{2}=F_{2} \sin \left(\phi_{2}\right) .
\end{aligned}
$$


Analytical and Numerical Investigation on the Duffing Oscilator...

and the differential equation becomes:

(13) $\ddot{x}+2 \gamma \dot{x}+\alpha x+\beta x^{3}=C_{1} \cos (p t)-S_{1} \sin (p t)+C_{2} \cos (n p t)-S_{2} \sin (n p t)$

For the aim of this investigation, it is needed to find polyharmonic solution in the form:

$$
x=A_{1} \cos (p t)+A_{2} \cos (n p t) .
$$

After substituting this solution in Eq. (13), one can obtain:

$$
\begin{aligned}
& \ddot{x}=-\beta\left[A_{1} \cos (p t)+A_{2} \cos (n p t)\right]^{3}-\alpha\left[A_{1} \cos (p t)+A_{2} \cos (n p t)\right] \\
&-2 p \gamma\left[-A_{1} \sin (p t)\right.\left.-n A_{2} \sin (n p t)\right]+C_{1} \cos (p t) \\
&-S_{1} \sin (p t)+C_{2} \cos (n p t)-S_{2} \sin (n p t) .
\end{aligned}
$$

Then, applying the following trigonometric relation:

$$
\cos ^{3}(a)=\frac{3}{4} \cos (a)+\frac{1}{4} \cos (3 a),
$$

Eq. (15) becomes:

$$
\begin{aligned}
\ddot{x}= & -\frac{3}{4} \beta A_{1}^{3} \cos (p t)-\frac{1}{4} \beta A_{1}^{3} \cos (3 p t) \\
& -3 \beta A_{1}^{2} A_{2} \cos ^{2}(p t) \cos (n p t)-3 \beta A_{1} A_{2}^{2} \cos (p t) \cos ^{2}(n p t) \\
& -\frac{3}{4} \beta A_{2}^{3} \cos (n p t)-\frac{1}{4} \beta A_{2}^{3} \cos (3 n p t)-\alpha A_{1} \cos (p t) \\
& -\alpha A_{2} \cos (n p t)+2 p \gamma A_{1} \sin (p t)+2 p \gamma n A_{2} \sin (n p t) \\
& +C_{1} \cos (p t)-S_{1} \sin (p t)+C_{2} \cos (n p t)-S_{2} \sin (n p t) .
\end{aligned}
$$

Now, one can use an elementary trigonometric relation and obtain:

(18) $\ddot{x}=-\frac{3}{4} \beta A_{1}^{3} \cos (p t)-\frac{1}{4} \beta A_{1}^{3} \cos (3 p t)$

$$
\begin{aligned}
& -\frac{3}{2} \beta A_{1}^{2} A_{2} \cos (n p t)-\frac{3}{2} \beta A_{1}^{2} A_{2}[\cos (2 p t) \cos (n p t)]-\frac{3}{2} \beta A_{1} A_{2}^{2} \cos (p t) \\
& -\frac{3}{2} \beta A_{1} A_{2}^{2}[\cos (p t) \cos (2 n p t)]-\frac{3}{4} \beta A_{2}^{3} \cos (n p t)-\frac{1}{4} \beta A_{2}^{3} \cos (3 n p t) \\
& -\alpha A_{1} \cos (p t)-\alpha A_{2} \cos (n p t)+2 p \gamma A_{1} \sin (p t)+2 p \gamma n A_{2} \sin (n p t) \\
& +C_{1} \cos (p t)-S_{1} \sin (p t)+C_{2} \cos (n p t)-S_{2} \sin (n p t)
\end{aligned}
$$


By applying the following basic trigonometric relation:

$$
\cos (a) \cos (b)=\frac{1}{2}[\cos (a+b)+\cos (a-b)],
$$

one can write:

$$
\begin{aligned}
& \ddot{x}=-\frac{3}{4} \beta A_{1}^{3} \cos (p t)-\frac{1}{4} \beta A_{1}^{3} \cos (3 p t)-\frac{3}{2} \beta A_{1}^{2} A_{2} \cos (n p t) \\
&-\frac{3}{4} \beta A_{1}^{2} A_{2}[\cos (n p t+2 p t)+\cos (n p t-2 p t)]-\frac{3}{2} \beta A_{1} A_{2}^{2} \cos (p t) \\
&-\frac{3}{4} \beta A_{1} A_{2}^{2}[\cos (2 n p t+p t)+\cos (2 n p t-p t)]-\frac{3}{4} \beta A_{2}^{3} \cos (n p t) \\
&-\frac{1}{4} \beta A_{2}^{3} \cos (3 n p t)-\alpha A_{1} \cos (p t)-\alpha A_{2} \cos (n p t)+2 p \gamma A_{1} \sin (p t) \\
&+2 p \gamma n A_{2} \sin (n p t)+C_{1} \cos (p t)-S_{1} \sin (p t)+C_{2} \cos (n p t)-S_{2} \sin (n p t) .
\end{aligned}
$$

After double integration of Eq. 20, the analytical solution of the Eq. 4 is found, as follows:

$$
\begin{gathered}
\text { (21) } x=\left[\frac{3}{16} \frac{\beta}{p^{2}} A_{1}^{3}+\frac{3}{2} \frac{\beta}{p^{2}} A_{1} A_{2}^{2}+\frac{\alpha}{p^{2}} A_{1}-\frac{C_{1}}{p^{2}}\right] \cos (p t)+\left[\frac{1}{36} \frac{\beta}{p^{2}} A_{1}^{3}\right] \cos (3 p t) \\
+\left[\frac{3}{4} \frac{\beta}{n^{2} p^{2}} A_{2}^{3}+\frac{3}{2} \frac{\beta}{n^{2} p^{2}} A_{1}^{2} A_{2}+\frac{\alpha}{n^{2} p^{2}} A_{2}-\frac{C_{2}}{n^{2} p^{2}}\right] \cos (n p t) \\
+\frac{3}{4} \beta A_{1}^{2} A_{2}\left[\frac{1}{n^{2} p^{2}+4 p^{2}} \cos (n p t+2 p t)+\frac{1}{n^{2} p^{2}-4 p^{2}} \cos (n p t-2 p t)\right] \\
+\frac{3}{4} \beta A_{1} A_{2}^{2}\left[\frac{1}{4 n^{2} p^{2}+p^{2}} \cos (2 n p t+p t)+\frac{1}{4 n^{2} p^{2}-p^{2}} \cos (2 n p t-p t)\right] \\
+\left[\frac{1}{36} \frac{\beta}{n^{2} p^{2}} A_{2}^{3}\right] \cos (3 n p t)+\left[\frac{S_{1}}{p^{2}}-\frac{2 \gamma A_{1}}{p}\right] \sin (p t)+\left[\frac{S_{2}}{n^{2} p^{2}}-\frac{2 p \gamma A_{2}}{n p^{2}}\right] \sin (n p t) .
\end{gathered}
$$

From equation (21), one can see that there are harmonics with frequencies $p, 3 p, n p, 3 n p, n p+2 p, n p-2 p, 2 n p+p, 2 n p t-p$. The sideband frequencies $[n p+2 p, n p-2 p]$ and $[2 n p+p, 2 n p t-p]$ are an evidence for an amplitude modulation and may be used as an indicator for defect presence.

\section{Numerical solution}

It is necessary to choose values for model parameters to find a numerical solution. It is reasonable such values to be used of the multipliers $\alpha$ and $\beta$ in Eq. 4 that correspond to bearing's stiffness in machines. In this investigation is used 
$\alpha=5.42 e 4 \mathrm{~N} / \mathrm{mm}$ and $\beta=6.88 e 10 \mathrm{~N} / \mathrm{mm}^{3}$ according to [16]. The point mass value is assumed to be $m=10 \mathrm{~kg}$. Then, for the coefficient of linear viscous friction is used $\gamma=55 \mathrm{Ns} / \mathrm{mm}$, according to Eq. 7 and [24]. The excitation force amplitudes are assumed to be equal as follows: $F_{1_{0}}=F_{2_{0}}=2 \mathrm{kN}$.

To solve numerically the Eq. 4, in this work is used Livermore Solver for Ordinary Differential Equations (LSODE) [25], which is implemented in function NDSolve of Wolfram Language. The LSODE is composed of stiff and non-stiff methods which switch when stiffness is sensed. The solver uses Adams method for the non-stiff regions and a variable step Backward Difference Formula (Euler's method) for the stiff regions.

A preliminary test is conducted before proceeding to solve Eq. 4. To check the suitability of the linear viscous friction coefficient value that has been chosen, the following equation is solved first:

$$
\ddot{x}+2 \gamma \dot{x}+\alpha x+\beta x^{3}=F_{1} \text { PieceWise }(t)
$$

where:

$$
\operatorname{PieseWise}(t)= \begin{cases}1, & t<0.1 \\ 0, & t \geq 0.1\end{cases}
$$

The solution is plotted in Fig. 2, and one can make an expert evaluation that the observed decay time of 0.1 seconds is normal for machinery supports.

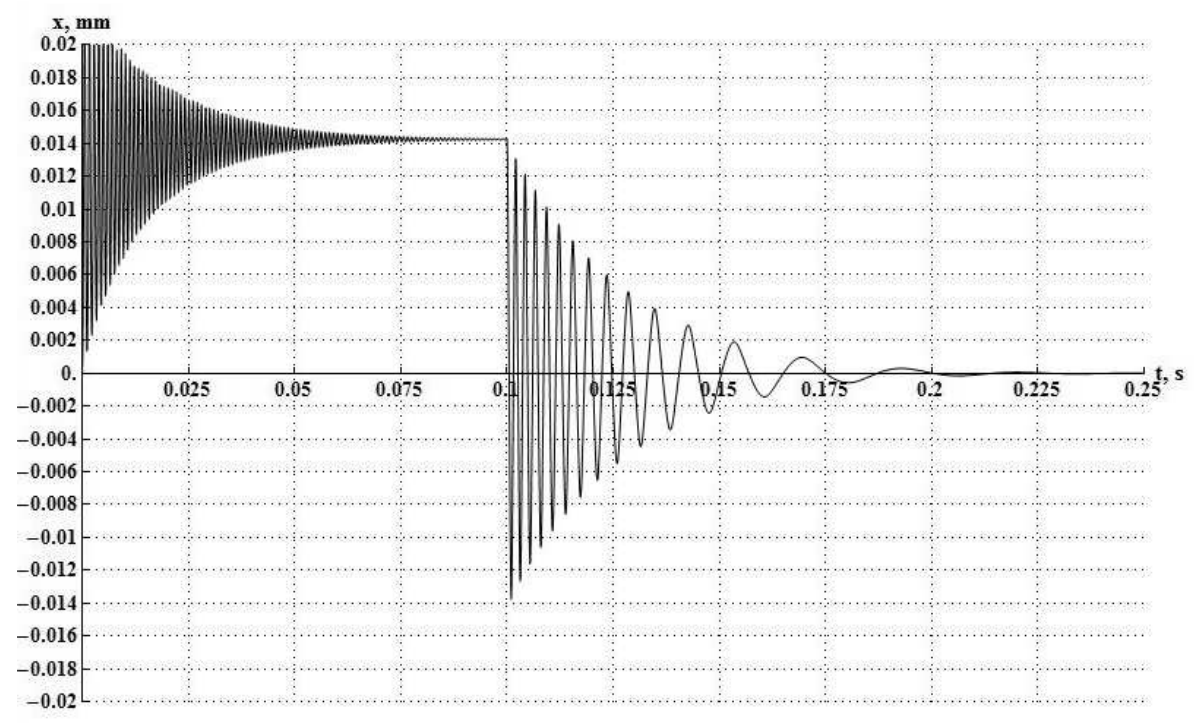

Fig. 2. Impulse response of the investigated system 


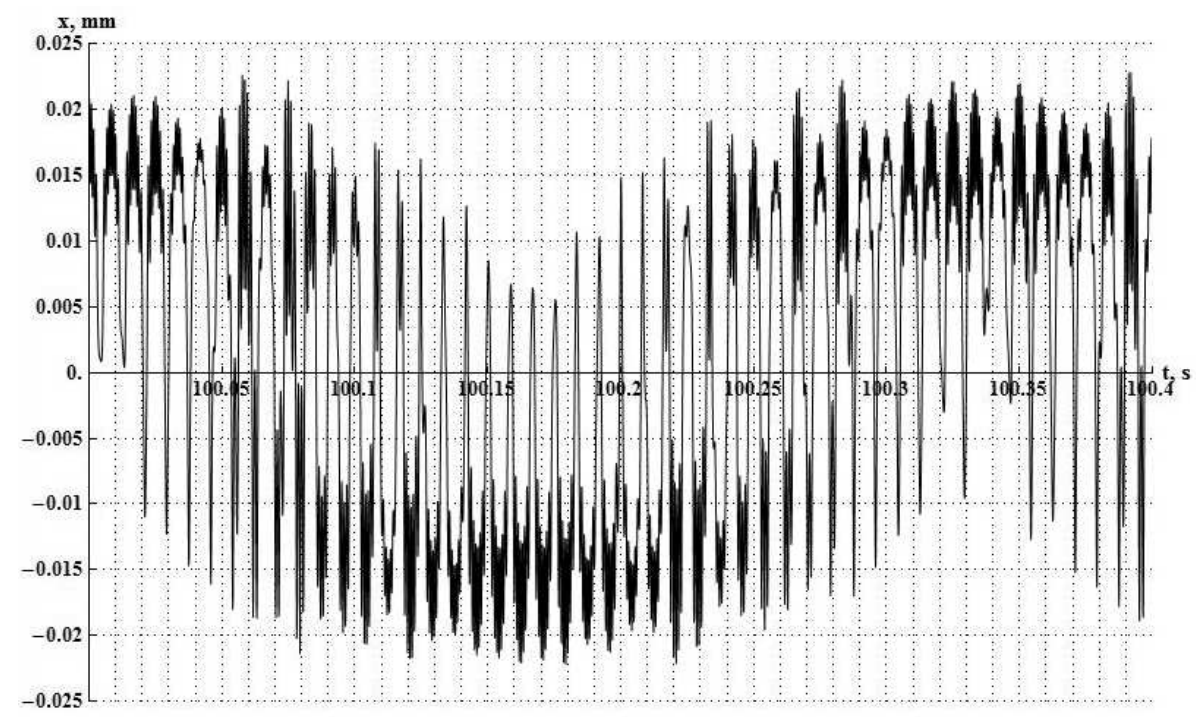

Fig. 3. Time diagram of the forced vibrations

Finally, the Eq. 4 is solved numerically for $\mathrm{p}=3 \mathrm{~Hz}$ and $\mathrm{n}=40$. Therefore, according to Eq. 21, in the results should be harmonics with frequencies:

$3,9,120,120+6,120-6,240+3,240-3$.

Through the solver used, results $x(t)$ in time domain are obtained, see Fig. 3. Then, they are transformed to frequency domain trough Discrete-time Fourier Transform (DFT):

$$
x(f)=D F T[x(t)]
$$

or

$$
x(f)=\frac{1}{\sqrt{n}} \sum_{t=1}^{n} x(t) e^{\frac{2 \pi i(f-1)(t-1)}{n}},
$$

where $n$ is the length of $x(t)$ and $f$ is the frequency in Hertz. The spectrograms obtained are presented in Figs 4 to 6 . One can observe a clear presence of the above mentioned harmonics. 


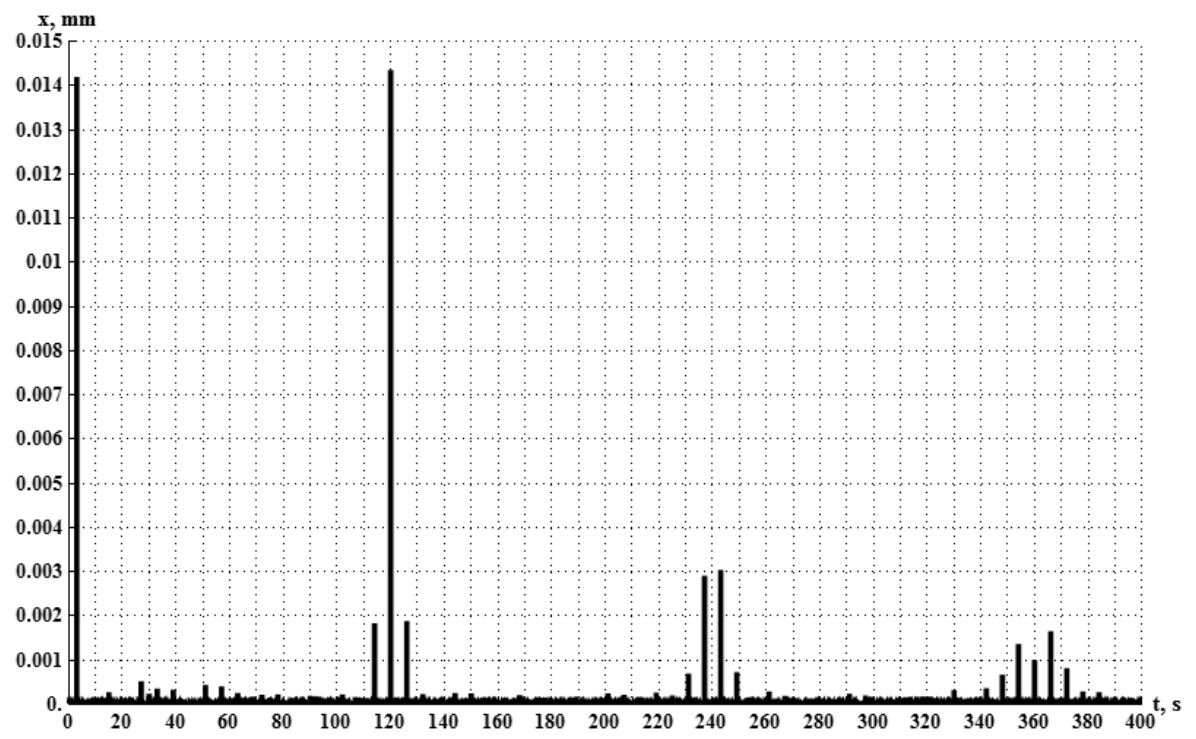

Fig. 4. Global view of the vibrations spectrogram

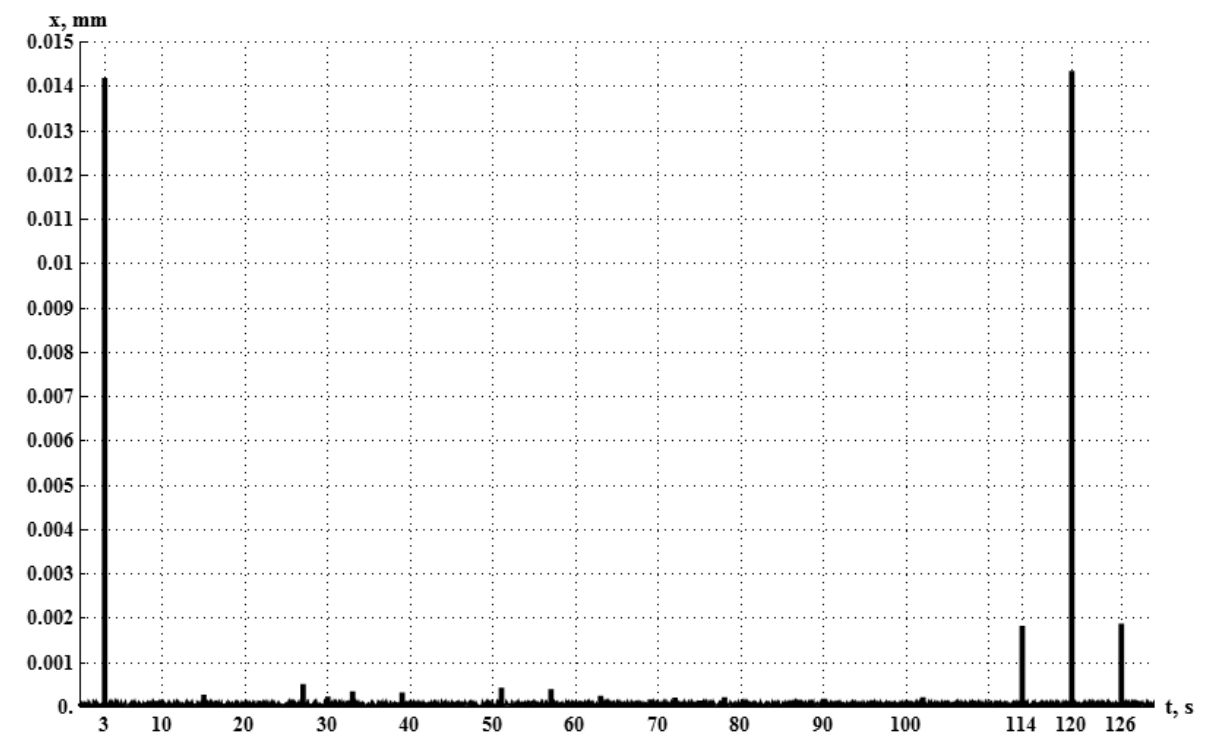

Fig. 5. The vibrations spectrogram, zoomed from 0 to $130 \mathrm{~Hz}$ 


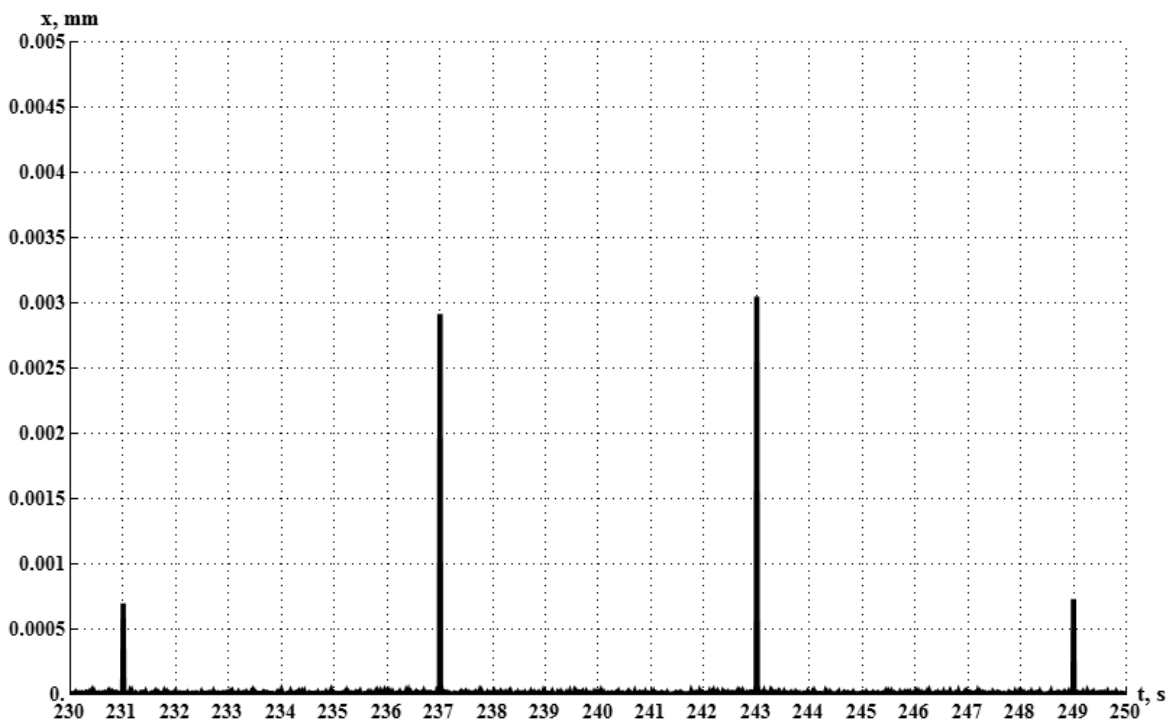

Fig. 6. The vibrations spectrogram, zoomed from 230 to $250 \mathrm{~Hz}$

\section{Analysis of the results}

The analytical solution proposed and the numerical one has similar results and show the presence of two phenomenon: frequency multiplication and amplitude modulation.

\subsection{Frequency multiplication}

In the results obtained possess harmonics with frequencies that are from a multiplication of excitation frequency with an integer. This may be explained by Eq. 16. One can write this equation as a function of time and with simple amplitude of two units, as follows:

$$
[2 \cos (22 \pi t)]^{3}=2^{3}\left[\frac{3}{4} \cos (22 \pi t)+\frac{1}{4} \cos (62 \pi t)\right],
$$

or:

$$
[2 \cos (22 \pi t)]^{3}=6 \cos (22 \pi t)+2 \cos (62 \pi t) .
$$

The left side of this equation is plotted in Fig. 7a. Then, DFT is performed and the spectrum obtained is shown in Fig. 7b. One can see two harmonics with following parameters:

- frequency $2 \mathrm{~Hz}$ and amplitude 5.4 units, 
- frequency $6 \mathrm{~Hz}$ and amplitude 1.9 units,

which frequencies are exactly as the frequencies in the right side of the equation and their amplitudes are approximately the same as the amplitudes in the right side of the equation, but a little differences exist because of the leakage, when the transform is performed.

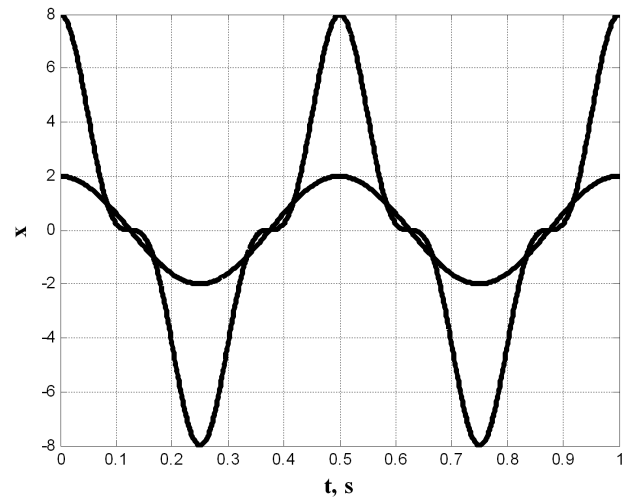

(a)

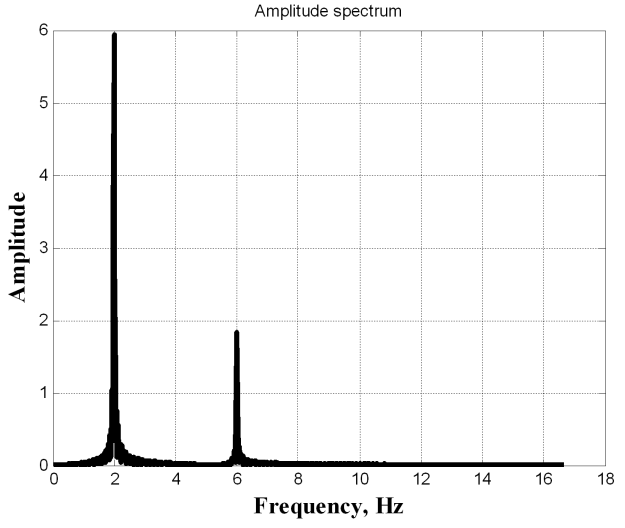

(b)

Fig. 7. Time (a) and frequency (b) illustration of a frequency multiplication due to raising a harmonic to 3 -rd power

\subsection{Amplitude modulation}

The analytical solution and the numerical one prove the existence of an amplitude modulation in vibration when the force excitation is polyharmonic unlike the case when the excitation force contains a single harmonic. From Eq. 21 and Figs 4 to 6 , one can see that the amplitude modulation that occurs has two sidebands at frequencies $[n p+2 p, n p-2 p]$ and $[2 n p+p, 2 n p t-p]$. Therefore, it is a Double Sideband Modulation (DSB) and is due to relation described by Eq. 19. One can write this equation in function of time and assume exemplary amplitude of two units, as follows:

$$
\begin{aligned}
2 \cos (22 \pi t) 2 \cos (10 & 2 \pi t) \\
& =\frac{1}{2}[2 \cos ((10+2) 2 \pi t)+2 \cos ((10-2) 2 \pi t)] .
\end{aligned}
$$

The left side of this equation is plotted in Fig. 8a. One can see that there is s polyharmonic signal as is in the right side of the equation. The result from DFT is shown in Fig. 8b. One can see the following sidebands: 


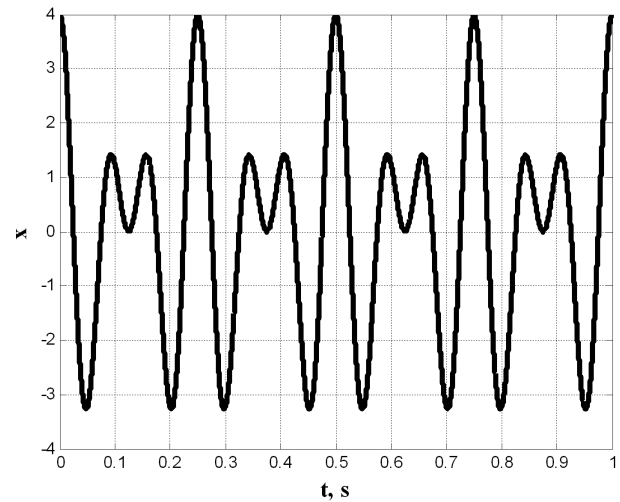

(a)

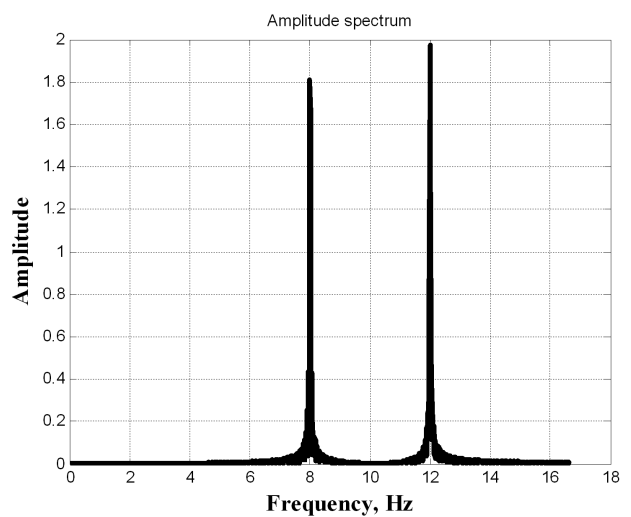

(b)

Fig. 8. Time (a) and frequency (b) illustration of an amplitude modulation due to multiplying of two harmonics with significant frequencies difference

- frequency $8 \mathrm{~Hz}$, amplitude 1.84 units,

- frequency $12 \mathrm{~Hz}$, amplitude 1.95 units,

which frequencies are exactly as the frequencies in the right side of the equation and their amplitudes are approximately the same as the amplitudes in the right side of the equation, but a little differences exist because of the leakage when the transform is performed.

In other words, the amplitude modulation is described by the mathematical multiplication operator. Therefore, when many harmonics acts on a nonlinear stiffness, they are multiplied for difference with when they act on a linear stiffness and the superposition principle is valid.

\section{Conclusions}

The analytical solution obtained in this work explains the amplitude modulation occurrence, when a polyharmonic force excitation acts on a support element with nonlinear stiffness. This can give an indicator for defect presence. The numerical solution proves the analytical results.

This work not fully solves the investigated problem. This is due to two facts, concerning the force acting on the supports:

- the force acting on the supports is applied as an external excitation force. Actually, it is an internal force and depends simultaneously on the defect and the form of the supports parameters;

- this excitation force is constructed by only two harmonics. It is needed more harmonics in real machinery. 
The analytical approach may not be suitable for investigating of this, but there is a way to solve through finite element analysis. This traces the future path of this investigation.

\section{REFERENCES}

[1] JiA, S., I. Howard. Comparison of Localised Spalling and Crack Damage from Dynamic Modelling of Spur Gear Vibrations. Mechanical Systems and Signal Processing, 20 (2006), 332-349.

[2] Wu, S., M. J. Zuo, A. Parey. Simulation of Spur Gear Dynamics and Estimation of Fault Growth. Journal of Sound and Vibration, 317 (2008), 608-624.

[3] Chen, Z., Y. Shao. Dynamic Simulation of Spur Gear with Tooth Root Crack propagating Along Tooth Width and Crack Depth. Engineering Failure Analysis, 18 (2011), 2149-2164.

[4] Pedersen, N. L., M. F. Jorgensen. On Gear Tooth Stiffness Evaluation. Computers and Structures, 135 (2014), 109-117.

[5] Feldman, M. Non-linear System Vibration Analysis using Hilbert Transform-I. Free Vibration Analysis Method 'Freevib', 8 (1994), No. 2, 119-127.

[6] Feldman, M. Non-linear System Vibration Analysis using Hilbert Transform-II. Forced vibration analysis method 'Forcevib', 8 (1994), No. 3, 309-318.

[7] Feldman, M. Considering High Harmonics for Identification of Non-linear Systems by Hilbert Transform. Mechanical Systems and Signal Processing, 21 (2007), 943-958.

[8] Feldman, M. Theoretical Analysis and Comparison of the Hilbert Transform Decomposition Methods. Mechanical Systems and Signal Processing, 22 (2008), 509-519.

[9] Feldman, M. Hilbert Transform in Vibration Analysis. Mechanical Systems and Signal Processing, 25 (2011), 735-802.

[10] Feldman, M. Hilbert Transform Applications in Mechanical Vibration, First Edition, Published 2011 by John Wiley \& Sons Ltd., ISBN: 978-0-470-97827-6.

[11] QU, L. L., L. X. LIAO. Haar Wavelet for Machine Fault Diagnosis. Mechanical Systems and Signal Processing, 21 (2007), 1773-1786.

[12] Li, H., Y. Zhang, H. Zheng. Application of Hermitian Wavelet to Crack Fault Detection in Gearbox. Mechanical Systems and Signal Processing, 25 (2011), 1353-1363.

[13] Combet, F., L. Gelman, G. LaPayne. Novel Detection of Local Tooth Damage in Gears by the Wavelet Bicoherence. Mechanical Systems and Signal Processing, 26 (2012), 218-228.

[14] Rafiee, J., M. A. Rafiee, P. W. Tse. Application of Mother Wavelet Functions for Automatic Gear and Bearing Fault Diagnosis. Expert Systems with Applications, 37 (2010), 4568-4579. 
[15] Saravanan, N., K. I. Ramachandran. Fault Diagnosis of Spur Bevel Gear Box using Discrete Wavelet Features and Decision Tree Classification. Expert Systems with Applications, 36 (2009), 9564-9573.

[16] Tiwari, R. On-line Identification and Estimation of Non-linear Stiffness Parameters of Bearings. Journal of Sound and Vibration, 234 (2000), No. 5, 906-910.

[17] Enns, R., G. C. McGuire. Nonlinear Physics with Mathematica for Scientist and Engineers, Birkhauser Boston, 2001, ISBN 0-8176-4223-4.

[18] Kovacic, I., M. J. Brennan. The Duffing Equation: Nonlinear Oscillators and their Phenomena, Edited by Ivana Kovacic, Michael J. Brennan, 2011, ISBN 978-0-470-71549-9.

[19] Patel, V. N., N. Tandon, R. K. Pandey. Defect Detection in Deep Groove Ball Bearing in Presence of External Vibration using Envelope Analysis and Duffing Oscillator. Measurement, 45 (2012), 960-970

[20] Cveticanin, L., G. Mester, I. Biro. Parameter Influence on the Harmonically Excited Duffing Oscillator. Acta Polytechnica Hungarica, 11 (2014), No. 5, 145160.

[21] Li, C., L. QU. Applications of Chaotic Oscillator in Machinery Fault Diagnosis. Mechanical Systems and Signal Processing, 21 (2007), 257-269.

[22] Zhao, Z., X. Wang, X. Zhang. Fault Diagnosis of Broken Rotor Bars in Squirrel-Cage Induction Motor of Hoister Based on Duffing Oscillator and Multifractal Dimension, Hindawi Publishing Corporation, Advances in Mechanical Engineering, 2014, Article ID 849670.

[23] Shi, H., S. Fan, W. Xing, J. Sun. Study of Weak Vibrating Signal Detection based on Chaotic Oscillator in MEMS Resonant Beam Sensor. Mechanical Systems and Signal Processing, 50-51 (2015), 535-547.

[24] Miller, B., S. Howard. Identifying Bearing Rotor-Dynamic Coefficients Using an Extended Kalman Filter. Tribology Transactions, 52 (2009), 671-679, Society of Tribologists and Lubrication Engineers ISSN: 1040-2004.

[25] Description and Use of LSODE, the Livermore Solver for Ordinary Differential Equations, NASA Reference Publication 1327, Lawrence Livermore Nationai Laboratory Report UCRL-ID-113855, 1993. 